\title{
Short Communication: COVID-19-Pandemie und HNO
}

\author{
Autoren \\ H.-J. Welkoborsky' ${ }^{1}$ A. Dietz ${ }^{2}$, T. Deitmer ${ }^{3}$ \\ Institute \\ 1 Klinik für HNO-Heilkunde, Kopf-Halschirurgie, \\ Klinikum Nordstadt, Hannover \\ 2 Univ. HNO-Klinik, Leipzig \\ 3 Deutsche Gesellschaft für HNO-Heilkunde, Kopf- und \\ Halschirurgie
}

\author{
Bibliografie \\ DOI https://doi.org/10.1055/a-1168-0855 \\ Online-Publikation: 8.5.2020 \\ Laryngo-Rhino-Otol 2020; 99: 370-373 \\ (c) Georg Thieme Verlag KG, Stuttgart · New York \\ ISSN 0935-8943 \\ Korrespondenzadresse \\ Prof. Dr. Dr. H.-J. Welkoborsky \\ Klinik für HNO-Heilkunde, Kopf-Halschirurgie, \\ Klinikum Nordstadt, Haltenhoffstr. 41, 30167 Hannover \\ welkohno.hanno@t-online.de
}

\section{Vorbemerkungen}

Nach seinem erstmaligem Auftreten Ende 2019 in der chinesischen Stadt Wuhan hat sich das neuartige Coronavirus-19 relativ schnell weltweit verbreitet. Es ist derzeit in nahezu allen Ländern mit regional sehr unterschiedlicher Inzidenz nachgewiesen. Mit dem neuartigen Coronavirus-19-Infizierte können an der durch das Virus verursachten COVID-19 (Syn. Severe acute respiratory syndrome coronavirus 2 (SARS-CoV-2)) erkranken. Nach Auskunft des Robert-Koch-Institutes (RKI) sind am 29.04.2020 weltweit über 3 Mio. Infektionen mit dem Virus nachgewiesen, 216700 Infizierte sind im Verlaufe der Infektion verstorben, wobei anhand der Daten nicht zu differenzieren ist, ob die COVID-19-Infektion in jedem Fall die unmittelbare Todesursache darstellte, oder die Patienten zwar als Virusträger, aber aus anderer Ursache verstorben sind. Für Deutschland werden am 28.04.2020157641 Infizierte angegeben, was einer Inzidenz von 190/100 000 Einwohner entspricht. 6115 Patienten sind im Verlauf der Infektion verstorben, 112000 Patienten gelten als genesen, so dass sich eine Anzahl an aktiv Infizierten von derzeit etwa 40000 ergibt, was einer Inzidenz von aktiv Infizierten von ca. 50/100 000 entspricht [1]. Allerdings wird immer wieder auf eine mutmaßlich hohe Dunkelziffer hingewiesen, auch vor dem Hintergrund, dass viele COVID-19-Infizierte klinisch asymptomatisch, aber Virusüberträger sein können [2, 3]. Dies gilt in besonderem Maße auch für Kinder, die nicht oder nur sehr selten symptomatisch erkranken $[4,5]$.

Verschiedene Bevölkerungsgruppen konnten als besondere Risikogruppen für eine COVID-19-Erkrankung identifiziert werden. Hierzu werden, auch und gerade vor dem Hintergrund der Erfahrungen mit dem Infektionsverlauf in Italien, Spanien und Frankreich, ältere Patienten mit einem Alter von über 60 Jahren und vorerkrankte Patienten, z. B. mit Herz-Kreislauf-Erkrankun- gen, Diabetes mellitus, schweren Lungenerkrankungen, Immunsuppression oder Tumorerkrankungen gezählt [6-8].

Aufgrund von regierungsamtlichen Anordnungen und Aufforderungen seitens der Gesundheitsbehörden wurden in vielen HNO-Kliniken und Praxen in den letzten Wochen seit dem 15.03.2020 die Behandlung elektiver Patienten verschoben. Es wurden weitgehend nur Notfallbehandlungen und -Eingriffe sowie onkologische Behandlungen und -Eingriffe durchgeführt. Bereits früh im Rahmen der Infektionsausbreitung in Deutschland haben die Präsidien der Deutschen Gesellschaft für HNO-Heilkunde, Kopf- und Halschirurgie sowie des Deutschen Berufsverbandes der Hals-Nasen-Ohren-Ärzte dazu aufgerufen, die elektive Tätigkeit im HNO-Gebiet einzustellen. Dies war im Lichte der akuten und sehr dynamischen Pandemiesituation gerechtfertigt und konsequent. Es wurden hiermit auch Kontakte von Patienten zu den Praxen und Kliniken reduziert, was unter infektionsepidemiologischen Gesichtspunkten geboten erschien, sowie Kapazitäten für die Behandlung einer großen Anzahl an COVID-19-Patienten geschaffen. Wenn auch die Behandlungsdringlichkeit in der HNO-Heilkunde, Kopf- und Halschirurgie geringer ist als z. B. in der Behandlung von Herzinfarkten oder Schlaganfällen, so gibt es in der HNO-Heilkunde onkologische Erkrankungen, entzündliche Erkrankungen an Mittelohr, Tonsillen und Nasennebenhöhlen, die innerhalb von wenigen Wochen zur Behandlung kommen müssen. Solche „nicht ganz hoch- aber doch dringliche“ Erkrankungen werden nun seit mehreren Wochen aufgeschoben, was auch die Patienten verständlicherweise beunruhigt.

Der Verlauf der Anzahl von Neuinfektionen pro Tag, der ansteigenden Verdoppelungsdauer der Infiziertenanzahl, der ansteigenden Anzahl an Genesenen, der sinkenden Zahl an Weiterinfizierten (Basisreproduktionszahl R0) und der sich abflachenden Kurve an Todesfällen von mit SARS-CoV-2 infizierten Patienten in Deutschland geben Anlass zur Hoffnung, dass die Infektionswelle an Dyna- 
mik verloren hat, und die Pandemie als beherrschbarer eingeschätzt werden kann. Hinzu kommt, dass in vielen Regionen Deutschlands ausreichende Kapazitäten in Kliniken und Praxen sowohl zur Versorgung von COVID-19- als auch von nicht-COVID-19Patienten zur Verfügung stehen, so dass eine Überforderung des Gesundheitssystems in Deutschland derzeit nicht absehbar ist.

Vor diesem Hintergrund haben die Präsidien der Deutschen Gesellschaft für HNO-Heilkunde, Kopf- und Halschirurgie sowie des Deutschen Berufsverbandes der Hals-Nasen-Ohren-Ärzte Gedanken und Empfehlungen entwickelt, in welcher Form ein Weg zurück in die Normalität der Gesundheitsversorgung möglich, sicher, verantwortungsvoll und sinnvoll ist. Mit gleicher Perspektive veröffentlichte das Bundesgesundheitsministerium am 27.4.20 ein Papier mit Ratschlägen für eine angepasste Krankenhausorganisation. https://cdn.hno.org/media/Corona-Ticker/ Mitteilung_BMG_27.4.20.pdf. Hierbei wird davon ausgegangen, dass die Problematik der COVID-19-Infektionen den medizinischen Alltag noch einige Zeit bestimmen wird. Zwar liegen einige ermutigende Resultate über die potenzielle Wirksamkeit einiger Substanzen bei COVID-19 vor; allerdings wird es nach allgemeiner Einschätzung noch einige Zeit bis zur Zurverfügungstellung eines Impfstoffes oder einer wirksamen Therapie dauern [9].

\section{Besonderheit des HNO-Gebietes}

Für den HNO-Bereich ergeben sich bei COVID-19 mehrere Besonderheiten:

- Gefährdungen durch das Virusreservoir insbesondere in der Nasenhaupthöhle

- die klinischen Symptome und die Therapie von Erkrankungen im Kopf-Hals-Bereich

Bereits früh in der Pandemie wurden Berichte aus China publiziert, wonach sich das neuartige Coronavirus insbesondere im Bereich von Nase, Nasenrachenraum, Larynx und Trachea nachweisen lässt und sich hier anreichert, und zwar bereits einige Tage bevor Patienten klinisch symptomatisch werden $[10,11]$. Auch haben frühe Berichte, wonach insbesondere HNO-Ärzte und Mitarbeiter von HNO-Ärzten aufgrund dieser Tatsache eine erhöhte Erkrankungsrate und Todesrate aufweisen, für Besorgnis gesorgt [12]. Allerdings beziehen sich diese Berichte auf die Frühphase des Infektionsgeschehens, als noch keine wirksamen Maßnahmen des persönlichen Schutzes zur Verfügung standen, und die pathogenetischen Kenntnisse der Infektion noch sehr lückenhaft waren $[12,25]$. Seither wurde wiederholt in den Medien über eine höhere Infektions- und Sterblichkeitsrate bei HNO-Ärzten berichtet, insbesondere aus Italien, Spanien, USA und Ecuador. Diese Berichte haben bisher jedoch keinen Einzug in die wissenschaftliche Literatur gefunden. Häufig bezogen sie sich auch auf Fälle, in denen keine persönlichen Schutzmaßnahmen bei der Untersuchung und Therapie von COVID-19-Erkrankten ergriffen wurden.

Zu den klinischen Symptomen bei COVID-19 gehören Fieber, trockener (häufiger) oder produktiver (seltener) Husten, Abgeschlagenheit, Müdigkeit, Gliederschmerzen und gastrointestinale Symptome ( $\triangleright$ Tab. 1). Symptome im Kopf-Hals-Bereich sind demgegenüber bisher zwar seltener berichtet worden, aber im Ergeb-
- Tab. 1 Übersicht über die häufigsten Symptome bei COVID-19Patienten [12-15, 19-21, 23].

\begin{tabular}{|l|l|}
\hline Symptom & Häufigkeit \\
\hline Fieber & $43-98 \%$ \\
\hline „Trockener“ Husten & $68-82 \%$ \\
\hline Produktiver Husten mit Sputumbildung & $28-33 \%$ \\
\hline Müdigkeit/Abgeschlagenheit & $38-44 \%$ \\
\hline Gastrointestinale Symptome & $20 \%$ \\
\hline Halskratzen/Heiserkeit & $14-17 \%$ \\
\hline Nasale Obstruktion & $5 \%$ \\
\hline Rhinorrhoe & $4 \%$ \\
\hline Hyposmie/Anosmie & bis 50\% \\
\hline Hypogeusie/Ageusie & bis 50\% \\
\hline Diffuser Schwindel & kasuistisch \\
\hline Hörminderung & bisher 1-3\% \\
\hline
\end{tabular}

nis bleibt jedoch festzuhalten, dass zahlreiche Patienten Symptome wie Husten, Heiserkeit, Halsschmerzen und Rhinitis zeigen, weswegen sie bei HNO-Ärzten vorstellig werden [14, 15]. Neben diesen Symptomen gehören häufig auch Riech- und Schmeckstörungen und gelegentlich zentralnervöse Symptome zu den Initialsymptomen der Erkrankung [16, 17]. Auch wird der HNO-Arzt mit der Durchführung von nasalen Abstrichtests konfrontiert [18]. In den letzten Wochen wurden Berichte veröffentlicht, wonach gehäuft sensorineurale Hörstörungen bei COVID-19-Patienten oder diffuser Schwindel auftreten können, wobei die Hörstörung persistieren kann [14, 19-21]. Dieses überrascht bei der nachgewiesenen Neurotropie des Virus nicht $[16,17]$. Es ist auch zu erwarten, dass HNO-Ärzte in Kliniken, in denen COVID-19-Patienten behandelt und beatmet werden, mit der Frage der Tracheotomie konfrontiert werden [22], wobei die Indikation hierfür sehr eng zu stellen ist, da meist eine Langzeitbeatmung von länger als vier Wochen nicht notwendig ist. Wird eine Tracheotomie aus Gründen der besseren Bronchialpflege, der Sorge um laryngotracheale Stenosen oder mit der Perspektive eines schnelleren Weanings notwendig, ist darauf zu achten, dass eine geblockte Kanüle eingesetzt wird, sodass ein geschlossenes System entsteht und eine Aerosolbildung möglichst vermieden wird [22]. Diese Beschreibungen verdeutlichen, warum HNO-Ärzte und auch das Personal in HNO-Praxen und -Kliniken zu den besonderen Risikogruppen im Gesundheitswesen gezählt werden, was auch von dem Center for Disease Control and Prevention der USA (CDC) bestätigt wird [23].

In der Literatur sind allerdings nur relativ wenige Arbeiten zur besonderen Gefährdung von Gesundheitspersonal veröffentlicht [24, 25]. Fest steht jedoch, dass Untersuchungen und Behandlungen an Nase, Nasennebenhöhlen, Oro- und Hypopharynx, Larynx und Trachea und mit Einschränkung auch im Bereich des Mittelund Innenohres aufgrund der oben ausgeführten Viruskonzentration zu den anatomischen Risikogebieten gezählt werden müssen. Alle Behandlungen und Untersuchungen, bei denen es zu einer Aerosolbildung kommen kann, sollten vermieden oder auf ein un- 
bedingt notwendiges Mindestmaß reduziert werden. Hierzu zählen auch endoskopische Untersuchungen der Nase und des Kehlkopfes, die, wenn notwendig, unter topischer Anästhesie mit einem Gelanästhetikum durchgeführt werden sollten, um Niesen etc. zu verhindern [22, 26, 27]. Gleiches gilt für die operativen Maßnahmen, bei denen z. B. im endonasalen und mastoidalen Bereich mit einer Aerosolbildung bei der Anwendung von Bohr- und Spülsystemen zu rechnen ist, nicht jedoch bei der Anwendung eines Microdebriders [27, 28].

Nicht zu vernachlässigen sind die zahlreichen asymptomatischen Patienten mit COVID-19-Infektion, die die HNO-Praxen und -Kliniken aufgrund von anderen Beschwerden aufsuchen, sowie Kinder, die z. B. zur Adenotomie vorgestellt werden und asymptomatische Virusträger sein können.

Somit ist gerade für die HNO-Heilkunde als besonders gefährdetem Fachgebiet die Frage der „Normalität“ der Patientenversorgung speziell. Offizielle Regelungen sind hier bisher unseres Wissens kaum ergangen und man darf erwarten, dass diese ähnlich wie bei der Frage des Maskenschutzes der Bevölkerung und der Ladenöffnungen nicht bundeseinheitlich sein werden. Es ist zu erwarten, dass möglicherweise je nach Votum von Gesundheitsämtern, Kommunen, Regierungsbezirken, Ärztekammern, Bundes- oder Landesbehörden regionale oder lokale Besonderheiten gültig werden können. Diese behördlichen Aussagen werden dann fraglos eine höhere Verbindlichkeit haben, als die hier niedergelegten Gedanken. Oft wird ein Arzt auch an die Vorgaben eines Trägers einer Klinik oder Einrichtung formal gebunden sein.

Dabei sind Regelungen der Patientenversorgung außerhalb des akuten Notfalls in der Hals-Nasen-Ohrenheilkunde von großer Bedeutung: So haben beispielsweise die Operationen an Nase, Nasennebenhöhlen und Adenoiden einen Anteil von zwischen $25 \%$ und $35 \%$ an allen operativen Leistungen einer HNO-Klinik/ Abteilung. Zudem zählen die Operationen, z. B. an der Nasenscheidewand oder an den Adenoiden, zu den häufigsten operativen Eingriffen beim Menschen in Deutschland per se.

In anderen Ländern mit COVID-19-Erkrankungen sind bereits Empfehlungen für die nicht notfallmäßige chirurgische Therapie von Erkrankungen im Allgemeinen und im HNO-Bereich im Speziellen formuliert worden, so z. B. vom American College of Surgeons, der Stanford University oder den Fachgesellschaften der Anästhesiologie [22, 26, 29, 30]. In Deutschland sind einige Überlegungen von einzelnen Kliniken bekannt (z. B. Univ. HNO-Klinik Jena, Univ. HNO-Klinik Leipzig, HNO-Klinik Klinikum Nordstadt Hannover). Ausgehend von diesen Überlegungen haben die Präsidien der Deutschen Gesellschaft für Hals-Nasen-Ohrenheilkunde, Kopf- und Halschirurgie sowie des Deutschen Berufsverbandes der HNO-Ärzte „Handlungsempfehlungen für die HNO-Elektiv-/ nicht notfallmäßige Behandlung in Corona-Zeiten " formuliert, die online publiziert sind (www.hno.org/corona Ticker) und auf die besonders hingewiesen wird.

\section{Fazit}

Hals-Nasen-Ohrenärzte werden häufig mit COVID-19 konfrontiert, sowohl aufgrund der Symptome des Patienten im akuten Krankheitsfall und nach Abklingen der akuten Infektion, als auch bei den diagnostischen und therapeutischen Prozeduren. Da zu erwarten ist, dass die COVID-19-Erkrankungen noch längere Zeit auftreten werden, wird sich für HNO-Ärzte, aber auch für HNOPraxis- und Klinikpersonal bleibend eine höhere Infektionsgefährdung ergeben. Dies unterstreicht die Notwendigkeit der Einhaltung des konsequenten persönlichen Schutzes und der Einhaltung von Präventionsmaßnahmen.

Interessenkonflikt

Die Autorinnen/Autoren geben an, dass kein Interessenkonflikt besteht.

Literatur

[1] Robert-Koch-Institut. COVID-19 Fallzahlen in Deutschland und weltweit. www.rki.de, Aufruf am 29.04.2020, 10.15 Uhr

[2] Balakrishnan K, Schechtman S, Hogikyan ND et al. COVID-19 Pandemic: What Every Otolaryngologist-Head and Neck Surgeon Needs to Know for Safe Airway Management. Otolaryngol Head Neck Surg 2020. doi:10.1177/0194599820919751

[3] Cheng X, Liu J, Li N et al. Otolaryngology Providers Must Be Alert for Patients with Mild and Asymptomatic COVID-19. Otolaryngol Head Neck Surg 2020. doi:10.1177/0194599820920649

[4] Matava CT, Kovatsis PG, Summers JL et al. PeDI-Collaborative: Pediatric Airway Management in COVID-19 patients - Consensus Guidelines from the Society for Pediatric Anesthesia's Pediatric Difficult Intubation Collaborative and the Canadian Pediatric Anesthesia Society. Anesth Analg 2020. doi:10.1213/ANE.0000000000004872

[5] Parikh SR, Bly RA, Bonilla-Velez J et al. Sie: Pediatric Otolaryngology Divisional and Institutional Preparatory Response at Seattle Children's Hospital after COVID-19 Regional Exposure. Otolaryngol Head Neck Surg 2020. doi:10.1177/0194599820919748

[6] Torretta S, Gaini LM, Pignataro L. Why Italian ENT physicians should be aware of SARS-CoV-2. Acta Otorhinolaryngol Ital 2020. doi:10.14639/ 0392-100X-N0738

[7] Aghagoli G, Gallo Marin B, Soliman LB et al. Cardiac involvement in COVID-19 patients: Risk factors, predictors, and complications: A review. J Card Surg 2020. doi:10.1111/jocs. 14538

[8] Saibene AM, Allevi F, Biglioli F et al. Role and Management of a Head and Neck Department during the COVID-19 Outbreak in Lombardy. Otolaryngol Head Neck Surg 2020. doi:10.1177/0194599820917914

[9] Costanzo M, De Giglio MAR, Roviello GN. SARS-CoV-2: Recent Reports on Antiviral Therapies Based on Lopinavir/Ritonavir, Darunavir/Umifenovir, Hydroxychloroquine, Remdesivir, Favipiravir and Other Drugs for the Treatment of the New Coronavirus. Curr Med Chem 2020. doi:10.2174/ 0929867327666200416131117

[10] Zou L, Ruan F, Huang M et al. SARS-CoV-2 Viral load in upper respiratory specimens of infected patients. N Engl J Med 2020. doi:10.1056/ NEJMc2001737

[11] Lu D, Wang H, Yu R et al. Integrated infection control strategy to. Minimize nosocomial infection of corona virus disease 2019 among ENT healthcare workers. J Hosp Infect 2020. doi:10.1016/j. jhin.2020.02.2018

[12] Wang D, Hu B, Hu C et al. Clinical characteristics of 138 hospitalized patients with 2019 novel coronavirus infected pneumonia in Wuhan, China. JAMA - J Am Med Assoc 2020; 323: 1061-1069

[13] Adams JG, Walls RM. Supporting the Health Care Workforce During the COVID-19 Global Epidemic. JAMA 2020; 323 (15): 1439-1440. doi:10.1001/jama.2020.3972 
[14] Krajewska J, Krajewski W, Zub K et al. COVID-19 in otolaryngologist practice: a review of current knowledge. Eur Arch Otorhinolaryngol 2020. doi:10.1007/s00405-020-05968-y

[15] Lovato A, de Filippis C, Marioni G. Upper airway symptoms in coronavirus disease 2019 (COVID-19). Am J Otolaryngol 2020. doi:10.1016/j.amjoto. 2020.102474

[16] Asadi-Pooya AA, Simani L. Central nervous system manifestations of COVID-19: A systematic review. J Neurol Sci 2020; 413: 116832. doi:10.1016/j.jns.2020.116832

[17] Siordia JA Jr. Epidemiology and clinical features of COVID-19: A review of current literature. J Clin Virol 2020; 127: 104357. doi: 10.1016/j.jcv. 2020.104357

[18] Xiang F, Wang X, He X et al. Antibody Detection and Dynamic Characteristics in Patients with COVID-19. Clin Infect Dis 2020. doi:10.1093/ cid/ciaa461

[19] Sriwijitalai W, Wiwanitkit V. Hearing loss and COVID-19: A Note. Am J Otolaryngol 2020. doi:10.1016/j.amoto.2020.102473

[20] Trecca EMC, Gelardi M, Cassano M. COVID-19 and hearing difficulties. Am J Otolaryngol; doi:10.1016/j.amoto.2020.102496

[21] Mustafa MWM. Audiological profile of asymptomatic COVID-19 PCR positive cases. Am J Otolaryngol 2020. doi:10.1016/j.amjoto.2020. 102483

[22] Vukkadala N, Qian Z], Holsinger FC et al. COVID-19 and the otolaryngologist - preliminary evidence-based review. Laryngoscope 2020 doi:10.1002/lary.28672

[23] Centers for Disease Control and Prevention (CDC). Interim infection prevention and control recommendations for patients with confirmed coronavirus disease 2019 (COVID-19) or persons under investigation for COVID-19 in healthcare settings. https://www.cdc.gov/coronavirus/ 2019-ncov/infection-control/control-recommendations.html
[24] Richardson S, Hirsch JS, Narasimhan M et al. Presenting Characteristics, Comorbidities, and Outcomes Among 5700 Patients Hospitalized With COVID-19 in the New York City Area. JAMA, J Americ Med Assoc Published online April 22, 2020 doi:10.1001/jama.2020.6775

[25] Chow E], Schwartz NG, Tobolowsky FA et al. Symptom screening at illness onset of health care personel with SARS-CoV-2 Infection in Kong County, Washington. JAMA Americ Med Ass, Apr. 17, 2020, publiziert online: https://jamanetwork.com

[26] Givi B, Schiff BA, Chinn SB et al. Safety Recommendations for Evaluation and Surgery of the Head and Neck During the COVID-19 Pandemic. JAMA Otolaryngol Head Neck Surg 2020. doi:10.1001/jamaoto. 2020.0780

[27] Patel ZM, Fernandez-Miranda J, Hwang PH et al. Letter: Precautions for Endoscopic Transnasal Skull Base Surgery During the COVID-19 Pandemic. Neurosurgery 2020. doi:10.1093/neuros/nyaa125

[28] Workman AD, Welling DB, Carter BS et al. Endonasal instrumentation and aerosolization risk in the era of COVID-19: simulation, literature review, and proposed mitigation strategies. Int Forum Allergy Rhinol 2020. doi: 10.1002 /alr.22577

[29] American College of Surgeons (ACS). Local Resumption of Elective Surgery Guidance. Publ. Online (abgerufen 22.04.2020): www.FACS.org

[30] Dexter F, Parra MC, Brown JR et al. Perioperative COVID-19 Defense: An Evidence-Based Approach for Optimization of Infection Control and Operating Room Management. Anesth Analg 2020. doi:10.1213/ ANE.0000000000004829.visual abstract icon 